\title{
Results on prognostic value of mutations in localized gastrointestinal stromal tumors (GIST) in one single center
}

\author{
Marina Garcés-Albir ${ }^{1}$, Roberto Marti-Obiol ${ }^{1}$, Fernando López-Mozos ${ }^{1}$, Silvia Calabuig-Fariñas ${ }^{2}$, \\ Samuel Navarro-Ros ${ }^{2}$ and Joaquín Ortega-Serrano ${ }^{1}$
}

${ }^{1}$ Unit of Esophago-Gastric Surgery. Department of General and Digestive Surgery. ${ }^{2}$ Department of Pathology. Hospital Clínico Universitario. Valencia, Spain

\begin{abstract}
Introduction: to study the prognostic value of mutations in KIT or PDGFRA in gastrointestinal stromal tumors (GIST) managed in our department.

Materials and methods: forty five patients with localized GIST underwent surgery between 1998 and 2010. Thirty six patients were enrolled in a retrospective study. DNA was isolated from 3 to $5 \mu \mathrm{m}$ sections of fixed and paraffin-embedded tissue. Exon 9, 11, 13 and 17 of c-kit gene and exon 12 and 18 of PDGFRA were amplified by PCR and sequenced.

Results: tumors with mutations were larger at the surgery and showed higher mitotic count $(p<0.05)$. The mutations were found in 22 patients $(61.2 \%), 18$ had mutations in exon 11 of c-kit gene. PDGFRA mutations were located in exon 12 . The 5-years relapsefree survival rate for patients with tumors having mutations was 38\% and $100 \%$ for patients without mutations $(\mathrm{p}<0.01)$. The 5 -year survival rate was significantly worse for patients with mutations $(20$ vs. 97\%, p < 0.01), with tumors larger than $5 \mathrm{~cm}(28$ vs. 97\%, p $<0.01$ ) and with $>50$ mitosis/HPF (42 vs. 88\%, p < 0.03). Multivariate analyses indicated that the mutations, mitotic counts, and tumor size were independent prognostic factors for survival in patients with localized GIST.

Conclusions: in this series, having a detected mutation is a poor prognostic factor with significantly increased recurrence rate and shortens survival.
\end{abstract}

Key words: Gastrointestinal stromal tumors (GIST). c-kit. PDGFRA. Mutation.

Some of these results were presented in a session of the XXVIII Annual National Congress of the Spanish Society of Surgeons, held in Madrid (November, 2010).

Received: 01-03-2012

Accepted: 29-05-2012

Correspondence: Marina Garcés Albir. Unit of Esophago-Gastric Surgery. Department of General and Digestive Surgery. Hospital Clínico Universitario. Avd. Blasco Ibañez, 17. 46010 Valencia, Spain

e-mail: garalma@hotmail.com

\author{
ABBREVIATIONS \\ PDGFRA: platelet-derived growth factor receptor alpha. \\ GIST: gastrointestinal stromal tumor. \\ PCR: polymerase chain reaction. \\ HPF: high power fields. \\ PET: positron emission tomography.
}

\section{INTRODUCTION}

Gastrointestinal stromal tumors (GISTs) are the most common mesenchymal tumors of the gastrointestinal tract. They are defined here as $c$-kit or KIT (CD117, stem cell factor receptor)-positive mesenchymal spindle cell or epithelioid neoplasm primary in the GI tract, omentum, mesentery or retroperitoneum (1).

Although around $87 \%$ of GIST expressed KIT, only 5792\% (according to the series and the diagnostic techniques) of GISTs showed mutations juxtamembrane to the $c$-kit gene domain (2-5).

The c-kit mutations are mainly located in the exons 9 , 11,13 and 17 . The frequencies of the mutations are $75-80 \%$ for the exon 11 and $20 \%$ for the exon 9 . Also we found mutations in another receptor, PDGFRA, principally in the exons 12 and 18 (6). An important characteristic in the molecular pathology of these tumors are that the c-kit and PDGFRA mutations are reciprocally excluded $(7,8)$.

There is a group of GIST (approximately 2-10\% of cases) that expresses very weakly or do not express c-kit, but in $10-50 \%$ of the cases they present c-kit or PDGFRA mutations and this helps to confirm the diagnosis of GIST (7).

Garcés Albir M, Marti R, López-Mozos F, Calabuig-Fariñas S, Navarro $S$, Ortega J. Results on prognostic value of mutations in localized gastrointestinal stromal tumors (GIST) in one single center. Rev Esp Enferm Dig 2012;104:405-410. 
The presence or absence of mutations in these tumors has predictive value for sensibility to treatment and prognostic value. Therefore, from the ESMO (European Society for Medical Oncology) the mutational analysis in the diagnostic work-up of all GIST is strongly recommended and it is required in CD117-negative cases when GIST is suspected. The centralization of mutational analysis in a laboratory enrolled in an external quality assurance program and with expertise in the disease in order to make mutational analysis more widely available is also proposed $(9,10)$.

The aim of this paper is to study the prognostic value of mutations in KIT or PDGFRA in GIST managed in our department.

\section{MATERIALS AND METHODS}

\section{Patients}

During the period 1998-2010, 45 patients with gastrointestinal stromal tumor were operated at the Unit of Esophago-Gastric Surgery, Department of General and Digestive Surgery, Hospital Clínico Universitario of Valencia, Spain. One GIST patient dropped out of the study after surgery, five patients had advanced disease (distant metastasis or peritoneal dissemination) at the time of diagnosis, and no good samples for DNA sequencing were obtained from three GIST patients. Therefore, a total of 36 patients were enrolled in this retrospective study. The locations of the tumors were as follows: 18 in the stomach, 13 in the small intestine, 1 in the large intestine, 1 in the rectum and 3 extraperitoneal. All cases were diagnosed by computed tomography (CT), and endorectal ultrasound in case of the rectal GIST. CT and sometimes PET allowed the preoperative staging. The patients with distant metastasis at the time of diagnosis or peritoneal dissemination were excluded from the study. The followup of these patients was physical exploration and abdominal CT every 6 months for two years and every year after. All patients with high risk or very high risk tumors, according to the Miettinen-Lasota (11) classification, were treated with Imatinib as from 2009. In this study, we analyzed the following data prospectively: age, sex, location, tumor size, histological type, mitotic count, mutations, recurrence and survival.

\section{Pathology}

Paraffin sections of formalin-fixed $(3 \mathrm{~m})$ were used for conventional hematoxylin and eosin staining and immunohistochemistry. The immunohistochemical analysis involved the StreptABComplex ${ }^{\circledR}$ (DAKO; Copenhagen, Denmark) method coupled to heat induced epitope retrieval, and the following antibodies were used: c-KIT (polyclonal, 1:400 dilution, DAKO; Copenhagen, Denmark), SMA(IA4 monoclonal, 1:2 dilution, DAKO), desmin (D33 monoclonal, 1:2 dilution, DAKO), S-100 (polyclonal, 1:2 dilution, DAKO), PDGFRA (polyclonal, 1:200 dilution, Santa Cruz Biotechnology; Madrid, Spain), Ki-67 (MIB-1 monoclonal, 1:50 dilution, DAKO), chromogranin (DAK-A3 monoclonal, 1:50 dilution, DAKO), CD99 (HO36-1.1 monoclonal, 1:50 dilution, DAKO), CD34 (Qbend-10 monoclonal, 1:50 dilution, DAKO), synaptophysin (SY38 monoclonal, 1:20 dilution, DAKO), vimentin (V9 monoclonal, 1:200 dilution, Novocastra Laboratories Ltd.; Newcastle, United Kingdom), p53 (DO-7 monoclonal, 1:400 dilution, DAKO) and MDM2 (IB10 monoclonal, 1:50 dilution, Novocastra). The histological variables analyzed for each tumor were as follows: mitotic count per 50 high-power fields (HPF) and predominant cell type (spindle, epithelioid or mixed).

\section{Molecular biology}

DNA was isolated from 3 to $5 \mathrm{~m}$ sections of fixed and paraffin-embedded tissue. After deparaffinization, DNA was extracted with a QUIAamp DNA FFPE Tissue Kit ${ }^{\circledR}$ (Qiagen; Venlo, Netherlands) and dissolved in 501 of ultrapure water. Intronic polymerase chain reaction (PCR) primers were used to amplify exons $9,11,13$ and 17 of $c$ KIT and exons 12 and 18 of PDGFRA. PCR was performed in a reaction volume of 501 containing 21 of DNA, 50 $\mathrm{mmol} / \mathrm{L} \mathrm{KCL}, 10 \mathrm{mmol} / \mathrm{L}$ Tris-HCL, pH 8.3,2 mmol/L $\mathrm{MgCl}_{2}, 0,2 \mathrm{mmol} / \mathrm{L}$ each dNTP, 0,2 $\mathrm{mol} / \mathrm{L}$ for each primer, and 2 units of AmpliTag Gold DNA Polimerase ${ }^{\circledR}$ (PerkinElmer; Norwalk, Conectica, USA). PCR was carried out in a DNA GeneAmp ${ }^{\circledR}$ System 9600 thermal cycler (PerkinElmer) after preheating the samples at $95^{\circ} \mathrm{C}$ for 10 minutes. The DNA was amplified over 40 cycler of: 1 minutes of denaturation at $94{ }^{\circ} \mathrm{C} ; 1,5$ minutes of annealing at $56{ }^{\circ} \mathrm{C}$ for $c$-KIT primers or 2 minutes at $65^{\circ} \mathrm{C}$ for $P D G F R A$ primers; and 1 minute of extension at $72{ }^{\circ} \mathrm{C}$ with an additional final extension step of 10 minutes. Ten microliters of the PCR products were visualized in ethidium-bromidestained 2\% UltraPure Agarose ${ }^{\circledR}$ gels (Life Technologies; Paisley, Scotland) and photographed. Negative controls were included in every set of amplifications. Bidirectional sequencing with specific primers was performed on an $\mathrm{ABI}$ PRISM $^{\circledR} 310$ sequencer using the BigDye ${ }^{\mathrm{TM}}$ Terminator V1.1 kit (Applied Biosystems; Foster City, California, USA).

\section{Statistical method}

Fisher's exact test, 'test, Student's $t$ test, and KaplanMeier method for postoperative survival with log rank test were used for statistical comparisons. Multivariate analysis was performed by Cox regression. Two-sided $P$ s of 0.05 was considered to represent statistical significance. The statistical comparisons were made with SPSS 15.0 to Windows (SPSS; Chicago, Illinois, USA). 
Table I. Tumors characteristics according to the presence or absence of mutation $(n=36)$

\begin{tabular}{lccc}
\hline & $\begin{array}{c}\text { No mutation } \\
(n=14)\end{array}$ & $\begin{array}{c}\text { Mutation } \\
(n=22)\end{array}$ & $p$ \\
\hline Age & 69.6 & 65.7 & 0.169 \\
Sex & & 12 & 0.441 \\
$\quad$ Male & 8 & 10 & \\
$\quad$ Female & 6 & 8.7 & 0.023 \\
Tumor size (cm) & 4.8 & 18 & 0.240 \\
Histological type & & 1 & \\
$\quad$ Spidle & 9 & 3 & \\
$\quad$ Epitheloid & 3 & & 0.345 \\
$\quad$ Mixed & 2 & 10 & \\
Tumor localization & & 9 & \\
$\quad$ Gastric & 8 & 0 & \\
$\quad$ Small Intestine & 4 & 2 & \\
Rectum & 1 & 1 & \\
$\quad$ Retroperitoneum & 1 & & \\
$\quad$ Colon & 0 & 14 & \\
Mitotic count (/50 HPF) & & 5 & \\
$\quad<5$ & 12 & 3 & \\
$\quad$ 6-10 & 2 & & \\
$>$ 10 & 0 & & \\
\hline
\end{tabular}

\section{RESULTS}

Of the 36 patients with localized GIST, 20 were males and 16 were females, with a median age of 67 years (range, 35-81 years). The most common site of the primary tumors was the stomach (18 cases) and small bowel (13 cases). The median tumor size was $5.6 \mathrm{~cm}$ (range, $2.3-12 \mathrm{~cm}$ ). Morphologically, 27 tumors $(75 \%)$ had the spindle cell type, 5 $(13.8 \%)$ had the mixed cell type and $4(11.1 \%)$ had pure epitheloid tumors. Tumors characteristics depending on the presence or absence of mutations were listed in table I. When we compared both groups, the tumors with mutations are larger at the time of surgery and showed higher mitotic

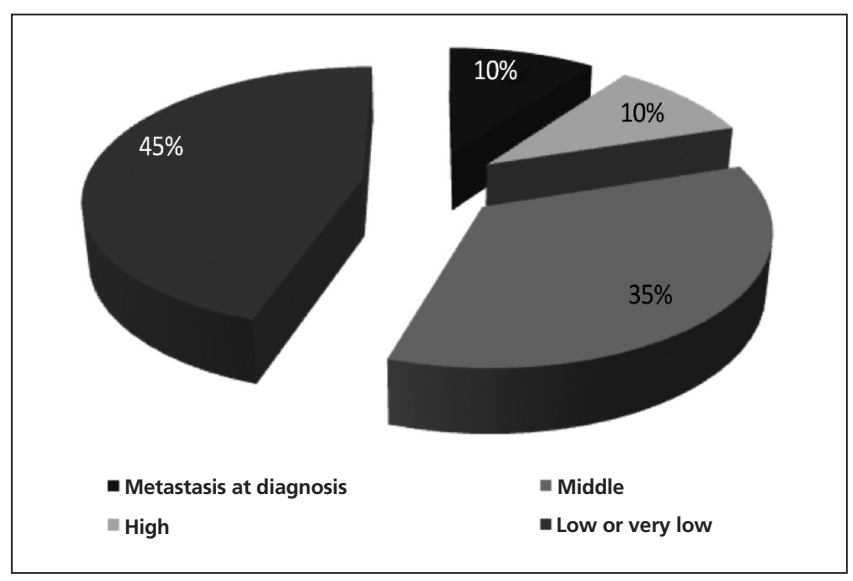

Fig. 1. Recurrence risk according to Miettinen-Lasota (9) $(n=36)$.
Table II. Summary of surgical techniques used $(n=36)$

\begin{tabular}{lcc}
\hline Surgical techniques & Laparotomy & Laparoscopic \\
\hline Total gastrectomy & 3 & \\
Atypical gastrectomy & 7 & 6 \\
Intragastric resection & & 2 \\
Intestinal resection & 8 & 5 \\
Right hemicolectomy & 1 & \\
Low rectal resection & 1 & \\
Retroperitoneal tumor resection & 3 & \\
\hline
\end{tabular}

counts. None of the other characteristics show significant differences between groups. The risk grade of the tumors, according to the Miettinen-Lasota classification (11), was very low or low risk in 16 patients $(44.4 \%)$ and only in 4 patients $(11.1 \%$ ) was very high risk (Fig. 1).

The surgical techniques used are summarized in table II. In the last 5 years, the surgery was carried out by laparoscopy when tumor size did not affect the principles of oncological surgery. No intraoperative tumor rupture occurred in any patients and all were $\mathrm{R} 0$ resection with a $0 \%$ postoperative mortality.

The c-kit or PDGFRA mutations were found in 22 patients (61.2\%), all was CD117 positive. Of the 22 GISTs with mutations, 18 had mutations in exon 11 and 2 in exon 9 of $c$-kit gene. None had mutations in exon 13 or 17 of ckit gene. PDGFRA mutations were located in exon 12. The most frequent amino acid alterations in the tumors were deletions ( 10 cases) and substitutions ( 9 cases) (Table III).

The median follow-up was 64.8 moths (range, 12-192 moths). Ten patients had recurrence of disease during follow-up time. The type of relapse was intra-abdominal in 6 cases, the liver in two cases and both sites in the other two cases. The 5-years relapse-free survival (RFS) rate for all patients was $68 \%$, whereas the overall survival rate was $76 \%$.

Table III. Distribution of mutations c-kit and PDGFRA ( $n=36)$

\begin{tabular}{lc}
\hline Mutation type & Number of patients (\%) \\
\hline No mutation & $14(38.8)$ \\
c-kit mutation & $20(55.5)$ \\
Exon 11 & $18(50)$ \\
Deletions & 7 \\
Sustitutions & 9 \\
Deletion insertion & 1 \\
Duplication & 1 \\
Exon 9 & $2(5.5)$ \\
Duplication & 2 \\
Exon 13 & $0(0)$ \\
Exon 17 & $0(0)$ \\
PDGFR mutation & $2(5.5)$ \\
Exon & \\
Deletions & 2 \\
\hline
\end{tabular}




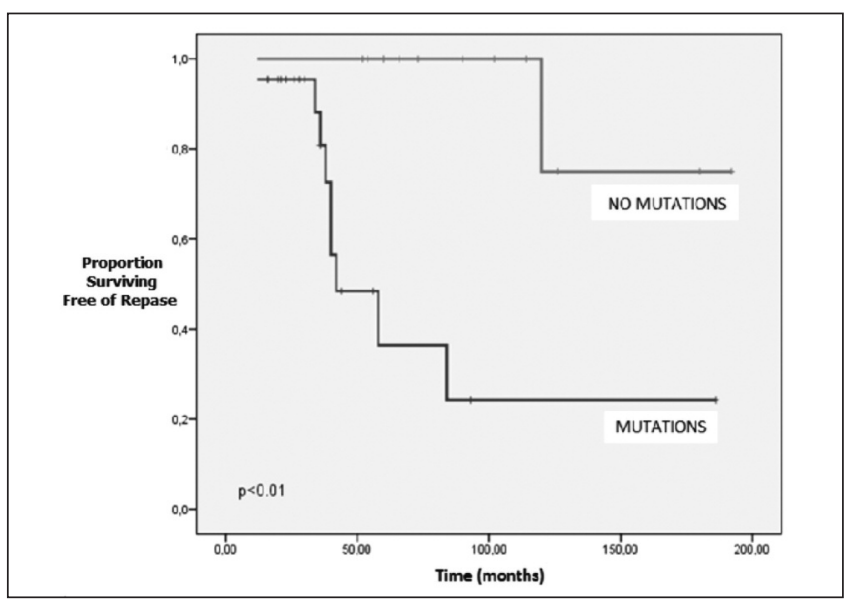

Fig. 2. The 5-years RFS between patients with GIST with or without mutations.

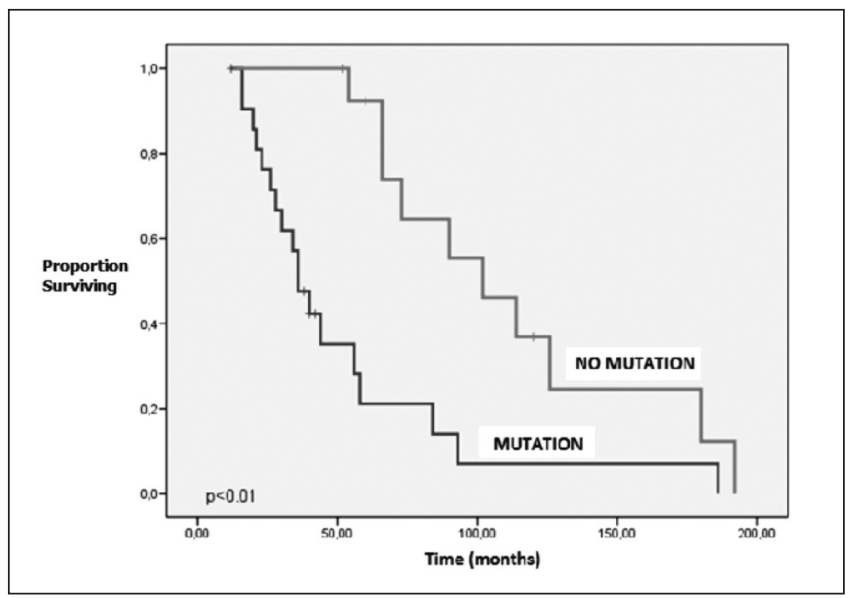

Fig. 3. Survival between GISTs with or without mutations.

The 5-years RFS rate for patients with tumors having mutations was 38\%, which was significantly lower than the 5years RFS for patients with tumors without mutations $(100 \%$, $\mathrm{p}<0.01$ ) (Fig. 2). We observed no statistically significant differences in the 5-year RFS with regards to the tumor size, tumor location, histological type and mitotic count.

The 5-year survival rate was significantly worse for patients with tumors having mutations (20 vs. 97\%, $\mathrm{p}<0.01$; Fig. 3), with tumors larger than $5 \mathrm{~cm}(28 v s .97 \%$,

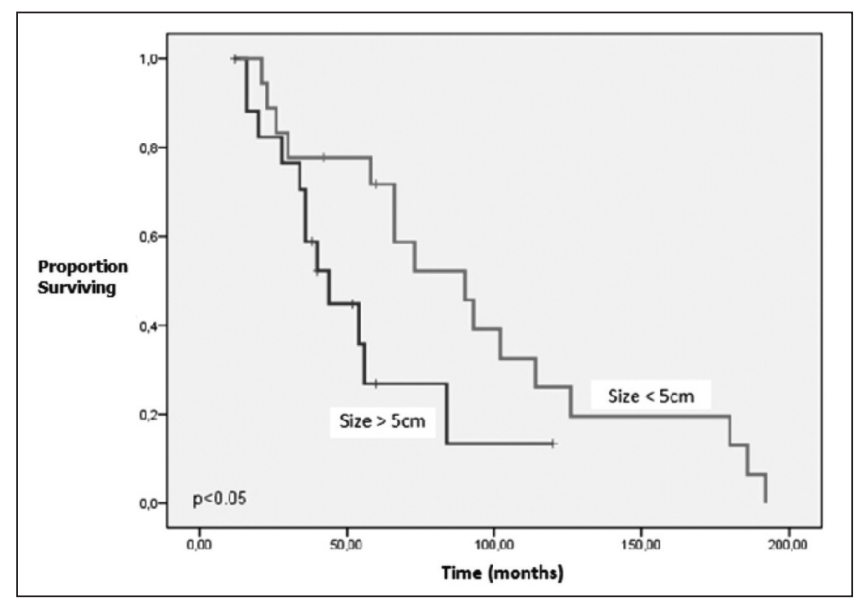

Fig. 4. Survival between GIST by tumor size.

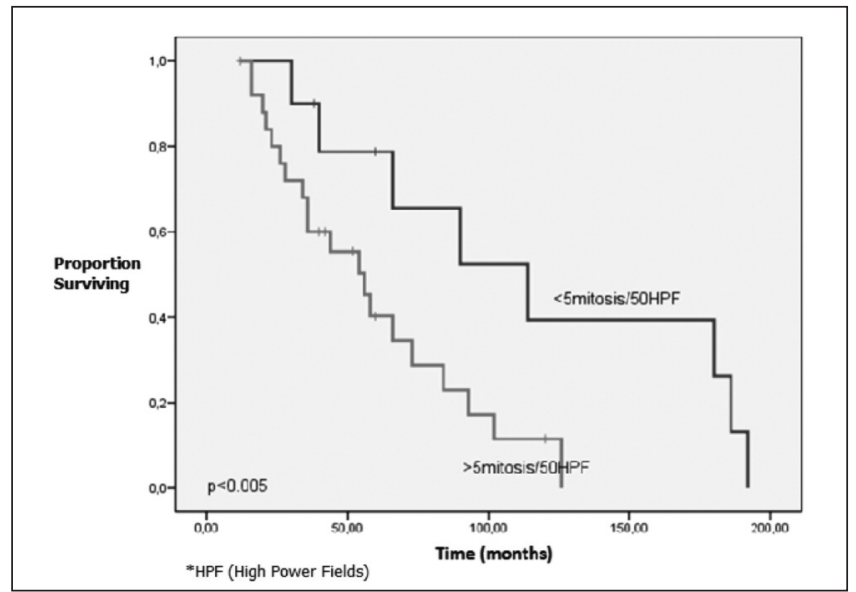

Fig. 5. Survival between GISTs by mitotic count.

$\mathrm{p}<0.01$; Fig. 4) and with more than 50 mitosis/HPF (High Power Fields) (42 vs. 88\%, p < 0.03; Fig. 5). Although the 5 -year survival rate was lower for patients with spindle GIST (47 vs. 73\%) and for no gastric GIST (45 vs. 59\%) also, were not found statistically significant differences.

Multivariate analyses indicated that the presence of mutations, mitotic counts, and tumor size was independent prognostic factors for survival in patients with localized GIST (Table IV).

Table IV. Multivariate analysis for survival

\begin{tabular}{lccc}
\hline Prognosis factors & Odds ratio & 95\% confidence interval & $p$ \\
\hline Presence of mutation & 27.6 & $2.18-48.1$ & 0.01 \\
$>5$ mitose $/ 50$ HPF & 2.2 & $1.48-10.3$ & 0.03 \\
Tumor size $>5$ cm & 7.8 & $1.73-82.82$ & 0.05 \\
Epitheloide or mixed type & 3.1 & $0.73-82.82$ & 0.25 \\
No gastric GIST & 2.3 & $0.47-20.70$ & 0.30 \\
\hline
\end{tabular}




\section{DISCUSSION}

The pathologic diagnosis of GIST relies on morphology and immunohistochemistry. Almost $90 \%$ of GIST are CD117 positive $(2,3)$. There is a proportion $(5 \%)$ of true GISTs which are CD117 negative and antigen retrieval may result in false CD117 staining. In these cases the mutational analysis plays an important role in discovering mutations involving c-kit and PDGFRA genes, because it can confirm the diagnosis of GIST, if doubtful $(12,13)$. In addition, mutational analysis has predictive value for sensitivity to imatinib therapy and prognostic implications (8). In our series, all cases were diagnosed by histology and immunohistochemistry, but the determination of the mutations had no diagnostic implications, because all of them were CD117 positive.

When we analyzed the different variables between patients with localized GIST with or without mutations, we observed that the patients with localized GIST who had mutations in the protooncogen c-kit or PDGFRA were associated with increased mitotic count or increased tumor size. Taniguchi et al. (2) also agree that there is a direct relationship between the presence of mutations in tumor size and mitotic count. The 5-years RFS and survival were also significantly lower in our patients with mutated GIST. However, we found no differences in 5-years RFS with tumor size, mitotic count or histological type.

The recurrence risk in GIST has been traditionally estimated by tumor size and mitotic counts (9). More recently, other factors have been included: tumor location (14), surgical margins, spontaneous or intraoperative tumor rupture (8) and the presence of mutations (3). Large tumor size remains the major predictor of recurrence rate (15). In this work we studied the prognostic significance of these factors and we found that tumors larger than $5 \mathrm{~cm}$ and the presence of more than 5 mitoses $/ 50$ HPF were clearly associated with worse outcome.

Multivariate analysis established independent factors for survival the presence of mutations, tumor size and mitotic count, in agreement with similar results published by other authors $(2,3,8,9)$. Although survival was also lower in patients with spindle tumors and no gastric location, we found no significant differences. In contrast, Miettinen et al. (10) and Demetri et al. (16) consider the tumor location as the basis for risk classification of these tumors. Our results can be explained by the limited number of patient included, not very high but all of them collected in one single center.

We can conclude that mutational analysis is a tool in the multidisciplinary management of GIST. In this series of patients, having a detected mutation was a poor prognostic factor with significantly increased recurrence rate and shortens survival.

In view of the results of this study and others, the mutation should be considered as a poor prognostic factor, and taken into account when managing possible neoadjuvant or adjuvant therapy. In both recent European and US guide- lines imatinib pretreatment is recommended if R0 surgery is not feasible and, at the moment, there is no global consensus but the adjuvant imatinib can be proposed as an option for patients with a substantial risk of relapse. In either case, mutational analysis may guide the selection of those patients, who are more likely to benefit from the treatment $(9,17)$.

Agreement with the others groups, including the Spanish Group for Sarcoma Research (GEIS), the prognostic value of mutations in GIST depends on the particular type of mutation (18). Therefore, we are working on a larger study to analyze the prognostic value of the different mutations. We continue to perform mutational analysis in our patients with GIST because we think it will be transcendental in the future to give good treatment and achieve better results.

\section{REFERENCES}

1. Miettinen M, Lasota J. Gastrointestinal stromal tumors definition, clinical, histological, inmunohistochemical, and molecular genetic features and differential diagnosis. Virchows Arch 2001;438:1-12.

2. Taniguchi M, Nishida T, Hirota S, Isozaki K, Ito T, Nomura T, et al. Effect of c-kit mutation on prognosis of Gastrointestinal Stromal Tumors. Cancer Res 1999;59:4297-300.

3. i Martín J, Poveda A, Llombart-Bosch A, Ramos R, López-Guerrero JA, García del Muro J, et al. Spanish Group for Sarcoma Research: Deletions affecting codons 557-558 of the c-kit gene indicate a poor pronognosis in patients with completely resected gastrointestinal stromal tumors: a study by the Spanish Group for Sarcoma Research (GEIS). J Clin Oncol 2005; 23:6190-8.

4. Kim TW, Lee H, Kang YK, Choe MS, Ryu MH, Chang HM, et al. Prognostic significate of c-kit mutation in localized gastrointestinal Stromal Tumors. Clin Cancer Res 2004;10:3076-81.

5. Singer S, Rubin BP, Lux ML, Chen CJ, Demetri GD, Fletcher CD, et al. Prognostic value of KIT mutation type, mitotic activity, and histologic subtype in Gastrointestinal Stromal Tumors. J Clin Oncol 2002;20:3898-905.

6. Miettinen M, Lasota J. Gastrointestinal Stromal Tumors. Review on morphology, molecular pathology, pronogsis, and differential diagnosis. Arch Pathol Lab Med 2006;130:1466-76.

7. Rubin BP, Heinrich MC, Corless CL. Gastrointestinal Stromal Tumour. Lancet 2007;369:1731-41.

8. Heinrich MC, Corless CL, Duensing A, McGreevey L, Chen CJ, Joseph $\mathrm{N}$, et al. PDGFRA activating mutations in gastrointestinal stromal tumors. Science 2003;299:708-10.

9. Casali PG, Jost L, Reichardt P, Schlemmer M, Blay JY. ESMO Guidelines Working Group (2009) Gastrointestinal stromal tumours: ESMO Clinical recommendation for diagnosis, treatment and follow up. Ann Oncol 2009;20:64-7.

10. Díaz-Delgado M, Hernández-Amate A, Sánchez-León M, PereiraGallardo S, Prieto-Sánchez E, Jiménez-Sáenz M, et al. Multiple nonmetastasic gastrointestinal stromal tumors. Differential features. Rev Esp Enferm Dig 2010;102:489-97.

11. Fletcher CD, Berman JJ, Corless C, Gorstein F, Lasota J, Longley BJ, et al. Diagnosis of gastrointestinal stromal tumours: a consensus approach. Human Pathol 2002;33:459-65.

12. Rader AE, Avery A, Wait CL, McGreevey LS, Faigel D, Heinrich MC. Fine-needle aspiration biopsy diagnosis of gastrointestinal stromal tumors using morphology, inmunocytochemistry, and mutational analysis of c-kit. Cancer 2001;93:269-75.

13. Scarpa M, Bertin M, Ruffolo C, Polese L, D’Amico DF, Angriman I. A systematic review on the clinical diagnosis of gastrointestinal stromal tumors. J Surg Oncol 2008;98:384-92.

14. Miettinen M, Lasota J. Gastrointestinal stromal tumours: pathology and prognosis at different sites. Semin Diagn Pathol 2006;30:477-89. 
15. Dematteo RP, Gold JS, Saran L, Gönen M, Liau KH, Maki RG, et al.Tumor mitotic rate, size and location independently predict recurrence after resection of primary gastrointestinal stromal tumor (GIST). Cancer 2008;112:608-15.

16. Demetri GD, Benjamin RS, Blanke CD, Blay JY, Casali P, Choi H, et al. NCCN Task Forceet (2007) NCCN Task Force report: management of patients with gastrointestinal stromal tumor (GIST) - update of the NCCN clinical practice guidelines. J Natl Compr Canc Netw 2007;5(Supl. 2):S1-29;quiz S30.
17. NCCN. The NCCN soft tissue sarcoma clinical practice guidelines in oncology (versión 1, 2009). (02009 National Comprehensive Cancer Network, Inc. Available at: http://www.ncen.org.

18. Martín-Broto J, Gutiérrez A, García-del-Muro X, López-Guerrero JA, Martínez-Trufero J, de Sande LM, et al. Prognosis time dependence of deletions affecting codons 557 and/or 558 of KIT gene for relapse-free survival (RFS) in localized GIST: a Spanish Group for Sarcoma Research (GEIS) study. Ann Oncol 2010;21:1552-7. 